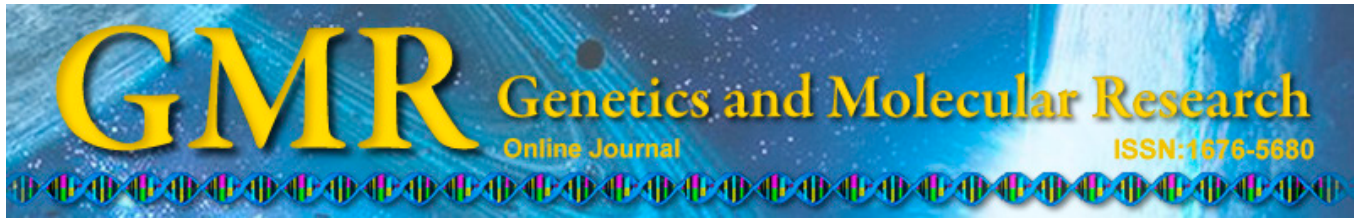

\title{
Relationship between serum GAD-Ab and the genetic polymorphisms of $G A D 2$ and type 2 diabetes mellitus
}

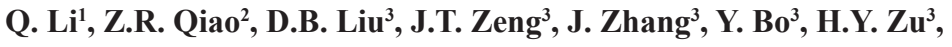 \\ Q. $\mathrm{Hu}^{2}, \mathrm{X} . \mathrm{Wu}^{2}$ and S.S. Dong ${ }^{3}$ \\ ${ }^{1}$ Department of Laboratory Medicine, \\ People's Hospital of Changshou, Chongqing, China \\ ${ }^{2}$ Department of Endocrinology, People's Hospital of Changshou, Chongqing, \\ China \\ ${ }^{3}$ Department of Laboratory Medicine, \\ People's Hospital of Changshou, Chongqing, China \\ Corresponding author: Q. Li \\ E-mail: qlzrcn@126.com
}

Genet. Mol. Res. 14 (2): 3002-3009 (2015)

Received March 17, 2014

Accepted July 4, 2014

Published April 10, 2015

DOI http://dx.doi.org/10.4238/2015.April.10.10

\begin{abstract}
In this study, we investigated the relationship between serum glutamic acid decarboxylase (GAD) autoantibody (Ab) levels and single nucleotide polymorphisms (SNPs) of the glutamic acid decarboxylase 2 (GAD2) 5'-untranslated region and the susceptibility to type 2 diabetes in the Han population. The distributions of patients with SNPs in the GAD2 5'-untranslated region (rs2236418, rs185649317, and rs8190590) and type 2 diabetes and that of the healthy group were genotyped and analyzed using Sequenom MassArray SNP genotyping. GAD-Ab levels were also detected. The frequency distributions of the AA, AG, and GG genotypes in the polymorphic site rs 2236418 in the diabetes GAD-Ab-positive group were 45.9, 42.8, and 11.4\%, respectively, whereas those in the control group were $36.6,43.7$, and $19.8 \%$, respectively. The difference between the 2 groups was statis-
\end{abstract}


tically significant $(\mathrm{P}<0.05)$. Unlike the GG genotype, the AA and $\mathrm{AA}+\mathrm{AG}$ genotypes increased the risk of GAD-Ab (odds ratios $(95 \%$ confidence intervals $)=2.623(1.351-4.937)$ and $2.152(1.375-4.202)$, respectively). The associations of the 3 SNPs of the GAD2 gene 5'-untranslated region polymorphisms with susceptibility to type 2 diabetes in the Chongqing Han population were significant. The SNP of rs2236418 in the Chongqing Han population of diabetic patients with serum GAD-Ab levels was significantly correlated with the SNPs rs 185649317 and rs 8190590 .

Key words: Diabetes mellitus; Glutamic acid decarboxylase 2; GAD-Ab; Single nucleotide polymorphism

\section{INTRODUCTION}

Diabetes is a lifelong disease with no cure. Type 2 diabetes mellitus (T2DM) is a typical multi-gene disease caused by several genetic factors (Scott et al., 2007; Yasuda et al., 2008; Li et al., 2013). A single factor poses little risk; the cumulative effect of several polymorphic loci increases the risk of T2DM. Hypoglycemic drugs and oral insulin are the primary means of controlling the blood sugar of T2DM patients. The illness occurs for a long duration during which islet dysfunction is severe, and secondary failure to sulfonylureas may occur. Even when the combined application of various oral hypoglycemic drugs does not effectively control blood sugar, insulin treatment must be continued for as long as possible to maintain the blood sugar at proper levels. The rational application of insulin can protect and restore cell functions. Functional lipids and coagulation and endothelial systems effectively control blood sugar and its induced disorders. The optimal administration time of insulin must be determined to effectively delay its subsequent complications and perform its important functions. Insulin release has an important function in the pathogenesis of T2DM (Genuth et al., 2003; Hansen et al., 2010). Glutamic acid decarboxylase (GAD) is the body's normal catalytic glutamic acid protease and gamma-aminobutyric acid involved in the synthesis and secretion of insulin from pancreatic $\beta$-cells. GAD is a key factor in the immune response for initiating the target antigen, specificity the inhibition caused by autoimmune reactions (Boutin et al., 2003) and induction of immune tolerance. GAD autoantibodies (GAD-Ab) can cause dysfunction of pancreatic $\beta$-cell autoimmunity (Kadiyala et al., 2010), which can gradually lead to islet $\beta$-cell failure. In cases, showing trends towards clinical manifestations of type 1 diabetes mellitus, insulin therapy is necessary (Prokopenko et al., 2008; Ali et al., 2011).

In this study, single nucleotide polymorphisms (SNPs) in the 5'-untranslated region (UTR) of the glutamic acid decarboxylase 2 (GAD2) gene's regulatory region were selected based on bioinformatic analysis. SNPs were selected from the dbSNP database in the 5'-UTR of GAD2 gene loci. Three SNP locus genotypes and serum GAD-Ab levels were detected in 497 patients and 500 normal controls. The optimal administration time of insulin therapy was determined based on personalized treatment data and theory by exploring the relationship between serum GAD-Ab level and GAD2 gene polymorphism (Johnson et al., 2002; Wheeler and Barroso, 2011). 


\section{MATERIAL AND METHODS}

\section{Specimens}

The blood samples from the 497 patients with T2DM and 500 normal controls were collected from the Changshou District People's Hospital from 2009 to 2011. The subjects were Han Chinese residents who lived in Chongqing and the surrounding areas, and they were mostly unrelated to one another. All cases included diagnosed clinical data. The control group recruited over the same period consisted of healthy volunteers. The case group consisted of 208 males and 289 females with an average age of $62.42 \pm 14.89$ years. The healthy control group consisted of 264 males and 236 females with an average age of $59.44 \pm 15.22$ years. After obtaining informed consent from the participants, $1-2 \mathrm{~mL}$ peripheral venous blood samples were collected and stored at $-70^{\circ} \mathrm{C}$. This study was conducted in accordance with the Declaration of Helsinki and with approval from the Ethics Committee of People's Hospital of Chongqing. Written informed consent was obtained from all participants.

\section{SNP loci}

The 3 SNP sites rs2236418, rs185649317, and rs8190590 specified in the www. snpedia.com database were located in the 5'-UTR region of the GAD2 gene. The Chinese population data in the HapMap database revealed the occurrence of polymorphisms.

\section{DNA extraction}

DNA was manually extracted from $200 \mu \mathrm{L}$ peripheral blood using a DNA extraction kit (Tiangen Biotechnology Co., Ltd., TIANamp Genomic DNA Kit, Beijing, China), and stored at $-70^{\circ} \mathrm{C}$.

\section{Primer design and synthesis}

For the gene target sequences of GAD2 and selected SNPs, the Sequenom MassArray Designer 3.0 software (Sand Diego, CA, USA) was used to design primers for polymerase chain reaction (PCR) amplification and extension of the 3 sites, including the specific amplification primer and extension sequences of TCTCCGCGCTTTCCTGAGTCCGGGC and TGGAGCAGGTCGCCTGCGAGTG, rs2236418 single base TAGTCCCGGTCTCTTTTAA, single base extension for rs 185649317 primer sequence CACGCGCGCGCAGGGCCAAGCC CGAG, and single base extension for rs8190590 primer sequence CAAGCCCGAGGCAGC TCGC. The primers were synthesized by Shanghai Invitrogen Corporation (Carlsbad, CA, USA).

\section{Genotyping analysis}

The SNP loci of the GAD2 gene promoter were genotyped using Sequenom MassARRAY mass spectrometry array technology.

After extracting and purifying the quantitatively diluted sample of genomic DNA, the resulting sample was placed on a 384-well plate. PCR amplification was performed using the following concentrations of reactants: $0.1 \mathrm{U}$ Taq polymerase, $5 \mathrm{ng}$ genomic DNA, 2.5 pmol 
of each PCR primer, and $2.5 \mathrm{mmol}$ dNTPs. The PCR conditions were as follows: $95^{\circ} \mathrm{C}$ for 15 min, 45 cycles at $95^{\circ} \mathrm{C}$ for $20 \mathrm{~s}, 56^{\circ} \mathrm{C}$ for $30 \mathrm{~s}$, and $72^{\circ} \mathrm{C}$ for $30 \mathrm{~s}$. The remaining dNTPs were removed by treatment with $0.3 \mathrm{U}$ shrimp alkaline phosphatase.

The single base extension reaction was conducted by adding 5.4 pmol extension primers, $50 \mu \mathrm{mol}$ ddNTP mixture, and $0.5 \mathrm{U}$ Thermosequenase DNA polymerase. The reaction conditions were as follows: $94^{\circ} \mathrm{C}$ for $2 \mathrm{~min}, 40$ cycles at $94^{\circ} \mathrm{C}$ for $5 \mathrm{~s}, 50^{\circ} \mathrm{C}$ for 5 $\mathrm{s}$, and $72^{\circ} \mathrm{C}$ for $5 \mathrm{~s}$. The reaction products were subjected to resin desalination for $20 \mathrm{~min}$ using a spotting instrument SpectroCHIP (Sequenom). After spotting, chip matrix-assisted laser desorption ionization time-of-flight mass spectrometry (SpectroREADER; Sequenom) was performed. DNA samples were evaluated for quality control and to ensure a genotyping success rate of $>95 \%$.

\section{Serum GAD-Ab}

The patients diagnosed with T2DM were subjected to drug therapy for $48 \mathrm{~h}$, after which the therapy was stopped. In the morning of the third day, 75 glucose was used (300 $\mathrm{mL}$ water) for the oral glucose tolerance test. The contents of the GAD-Ab protein chip for 0 min serum samples were obtained, and specific methods were performed according to manufacturer instructions.

\section{Statistical analysis}

The Hardy Weinberg equilibrium test was performed for both the case and control groups. Genotype frequencies were detected using the SPSS 13.0 statistical software (SPSS, Inc., Chicago, IL, USA). The chi-squared test was conducted to determine the differences in the allelic and genotype frequency distributions. GAD-Ab test results were used to classify subjects into GAD-Ab-positive and GAD-Ab-negative groups. The differences in the genotype frequency distribution of the SNP locus for chi-square analysis were determined. Nonconditional logistic regression was used to calculate the $\mathrm{P}$ values of different genotypes. The significance level was set at 0.05 .

\section{RESULTS}

The case group matched the control group for age; differences were not statistically significant $(\mathrm{P}>0.05)$. The frequency distributions of the 3 genotypes were in accordance with Hardy-Weinberg equilibrium in both groups $(\mathrm{P}>0.05)$.

\section{SNP site rs2236418}

The AA, AG, and GG genotypes in rs2236418 were detected in the Chongqing Han population. The frequency distributions for AA, AG, and GG in the case group were 40.8, 43.2 , and $15.9 \%$, respectively. The frequency distributions for AA, AG, and GG in the healthy control group were $37.4,45.8$, and $16.8 \%$, respectively. The differences were not significant $\left(\chi^{2}=0.312\right.$ and $\mathrm{P}=0.862$; Table 1$)$. 
Table 1. Relationship between rs2236418 genotype distribution and susceptibility to diabetes [N (\%)].

\begin{tabular}{|c|c|c|c|c|}
\hline Genotype of rs 2236418 & Case group & Control group & $\chi^{2}$ & $P$ \\
\hline$\overline{\mathrm{AA}}$ & $203(40.8)$ & $187(37.4)$ & \multirow{3}{*}{0.312} & \multirow{3}{*}{0.862} \\
\hline AG & $215(43.2)$ & $229(45.8)$ & & \\
\hline GG & $79(15.9)$ & $84(16.8)$ & & \\
\hline
\end{tabular}

For the GAD-Ab level of the case group, 229 cases showed positive results and 268 cases showed negative results. The frequency distributions of AA, AG, and GG in the GADAb-positive group were $45.9,42.8$, and $11.4 \%$, respectively. The frequency distributions of $\mathrm{AA}, \mathrm{AG}$, and $\mathrm{GG}$ in the GAD-Ab-negative group were $36.6,43.7$, and $19.8 \%$, respectively. The differences were statistically significant $\left(\chi^{2}=8.085\right.$ and $\mathrm{P}=0.013$; Table 2$)$.

Table 2. Relationship between rs2236418 genotype distribution and serum levels of GAD-Ab [N (\%)].

\begin{tabular}{lcccc}
\hline Genotype of rs2236418 & GAD-Ab-positive & GAD-Ab-negative & $\chi^{2}$ & P \\
\hline AA & $105(45.9)$ & $98(36.6)$ & & 0.013 \\
AG & $98(42.8)$ & $117(43.7)$ & $53(19.8)$ & \\
GG & $26(11.4)$ & & & \\
\hline
\end{tabular}

Logistic regression analysis, adjusted for gender and age, was performed to evaluate the level of correlation between rs2236418 and T2DM patients with GAD-Ab. Unlike the GG genotype, the AA and AA + AG genotypes increased the risk of GAD-Ab. The respective odds ratios (ORs) [95\% confidence interval (CI)] were $2.623(1.351-4.937)$ and 2.152 (1.375-4.202) (Table 3).

Table 3. Risk of diabetes related to rs2236418 genotype and GAD-Ab.
\begin{tabular}{lcccc}
\hline Genotype of rs2236418 & GAD-Ab-positive & GAD-Ab-negative & P & OR (95\%CI) \\
\hline GG & 26 & 53 & & 1 \\
AA & 105 & 98 & 0.005 & $2.623(1.351-4.937)$ \\
AG & 98 & 117 & 0.063 & $1.822(0.892-3.463)$ \\
AA+AG & 203 & 215 & 0.021 & $2.152(1.375-4.202)$ \\
\hline
\end{tabular}

\section{SNP site rs185649317}

The AA, AG, and GG genotypes in rs185649317 in the Chongqing Han population were determined. The frequency distributions of AA, AG, and GG in theT2-DM group were $6.6,39.4$, and $53.9 \%$, respectively. The frequency distributions of AA, AG, and GG in the healthy control group were $6.0,40.6$, and $53.4 \%$. The differences were not statistically significant $\left(\chi^{2}=0.651\right.$ and $P=0.967$; Table 4$)$.

Table 4. Relationship between rs185649317 genotype distribution and susceptibility to diabetes [N (\%)].

\begin{tabular}{|c|c|c|c|c|}
\hline Genotype of rs 185649317 & Case group & Control group & $\chi^{2}$ & $\mathrm{P}$ \\
\hline$\overline{\mathrm{AA}}$ & $33(6.6)$ & $30(6.0)$ & \multirow{3}{*}{0.651} & \multirow{3}{*}{0.967} \\
\hline AG & $196(39.4)$ & 203 (40.6) & & \\
\hline GG & $268(53.9)$ & $267(53.4)$ & & \\
\hline
\end{tabular}


The frequency distributions of $\mathrm{AA}, \mathrm{AG}$, and $\mathrm{GG}$ in the GAD-Ab-positive group were 7.0, 41.9, and $51.1 \%$, respectively. The frequency distributions of $\mathrm{AA}, \mathrm{AG}$, and $\mathrm{GG}$ in the GAD-Ab-negative group were $6.3,37.3$, and $56.3 \%$, respectively. The differences were not statistically significant $\left(\chi^{2}=0.621\right.$ and $\mathrm{P}=0.713$; Table 5$)$.

Table 5. Relationship between rs 185649317 genotype distribution and serum levels of GAD-Ab [N (\%)].

\begin{tabular}{|c|c|c|c|c|}
\hline Genotype of rs185649317 & GAD-Ab-positive & GAD-Ab-negative & $\chi^{2}$ & $\mathrm{P}$ \\
\hline$\overline{\mathrm{AA}}$ & $16(7.0)$ & $17(6.3)$ & \multirow{3}{*}{0.621} & \multirow{3}{*}{0.713} \\
\hline AG & 96 (41.9) & $100(37.3)$ & & \\
\hline GG & $117(51.1)$ & $151(56.3)$ & & \\
\hline
\end{tabular}

\section{SNP site rs8190590}

The CC, CT, and TT genotypes in rs8190590 of the Chongqing Han population were determined. The frequency distributions of CC, CT, and TT in the T2-DM group were 52.1, 36.4 , and $11.5 \%$, respectively. The frequency distributions of CC, CT, and TT in the control group were $44.4,39.4$, and $16.2 \%$, respectively. The differences were not statistically significant $\left(\chi^{2}=0.197\right.$ and $\mathrm{P}=0.232$; Table 6).

Table 6. Relationship between rs8190590 genotype distribution and susceptibility to diabetes [N (\%)].
\begin{tabular}{lrrrr}
\hline Genotype of rs8190590 & Case group & Control group & $\chi^{2}$ & P \\
\hline CC & $259(52.1)$ & $222(44.4)$ & 0.197 \\
CT & $181(36.4)$ & $197(39.4)$ & & 0.232 \\
TT & $57(11.5)$ & $81(16.2)$ & & \\
\hline
\end{tabular}

The frequency distributions of CC, CT, and TT in the GAD-Ab-positive group were $55.5,26.6$, and $17.9 \%$, respectively. The frequency distributions of CC, CT, and TT in the GAD-Ab-negative group were $49.3,44.8$ and $6.0 \%$, respectively. The differences were not statistically significant $\left(\chi^{2}=0.546\right.$ and $\mathrm{P}=0.641$; Table 7$)$.

Table 7. Relationship between rs8190590 genotype distribution and serum levels of GAD-Ab [N (\%)].

\begin{tabular}{lcccc}
\hline Genotype of rs8190590 & GAD-Ab-positive & GAD-Ab-negative & $\chi^{2}$ & P \\
\hline AA & $127(55.5)$ & $132(49.3)$ & & 0.546 \\
AG & $61(26.6)$ & $120(44.8)$ & & 0.641 \\
GG & $41(17.9)$ & $16(6.0)$ & & \\
\hline
\end{tabular}

\section{DISCUSSION}

GAD2 encodes GAD65, which is considered to be one of the most important islet autoantigens. GAD2 contains 1758 nucleotides, which encode for 585-amino acid residues (Kaufman et al., 1993). Small-scale clinical tests showed that GAD2 may delay latent autoimmune diabetes in adults with no significant side effects. GAD is primarily expressed in the brain and islet B cell-secretory granules. It occurs in 2 isoforms, GAD2 and GAD1, and is located on chromosomes 10 and 2; their non-allelic molecular weights are approximately 65 
and $67 \mathrm{kDa}$, and thus are often referred to as GAD65 and GAD67 (Boutin et al., 2003; Jayakrishnan et al., 2011). GAD65 and GAD67 share 65\% amino acid sequence identity. Their main difference lies in their amino termini in amino acids 325-355. In different types of animals, GAD is expressed in the islets. The human islet only contains GAD2 (Karlsen et al., 1991; Jun et al., 2002).

GAD has two isozymes, but mainly for GAD-Ab GAD2 (Jayakrishnan et al., 2011; Sosenko et al., 2011). After the onset of diabetes, GAD-Ab appears earlier than other autoantibodies and is present for a longer duration; the age span, positive rate, and other characteristics of GAD-Ab can be detected through the diagnosis of latent autoimmune diabetes in adults (Appel et al., 2009). Recent studies found that T2DM patients were positive for GAD-Ab; the viability of islet $\mathrm{T}$-cell responses during positive immunity has also been examined (BrooksWorrell et al., 2011).

In a case-control study involving 575 obese patients and 646 healthy subjects, Boutin et al. (2003) found that gene polymorphisms in GAD2 sites of the $61450 \mathrm{C}>\mathrm{A}$ and 83897 $\mathrm{T}>\mathrm{A}$ haplotypes had a protective effect on obesity $(\mathrm{OR}=0.81,95 \% \mathrm{CI}=0.681-0.972, \mathrm{P}=$ $0.0049)$, and confirmed the SNP $-243 \mathrm{~A}>\mathrm{G}$ obesity susceptibility locus $(\mathrm{OR}=1.3,95 \% \mathrm{CI}=$ $1.053-1.585, \mathrm{P}=0.014)$. The $3 \mathrm{SNPs}$ were significantly associated with GAD-Ab levels $(\mathrm{P}=$ $0.003,0.047$, and 0.006) (Davies et al., 2008). However, in a study examining the GAD2 gene polymorphism in type $1 \mathrm{DM}$, Johnson et al. (2002) selected SNP loci covering the GAD2 gene exon 3'-UTR, 5'-UTR, and 5'-upstream region, but failed to detect the GAD2 gene polymorphism in patients with type 1 DM in 2 selected European populations (Boutin et al., 2003; Hampe et al., 2007).

A case-control study was conducted that included 497 patients with T2DM and 500 normal controls. Patients were genotyped, and the SNP locus for the 3 genotypes of the GAD2 5'-UTR area was selected. However, no significant correlation between the GAD2 gene and susceptibility to T2DM was found in the Chongqing Han population. Further evaluation indicated that SNP rs2236418 was significantly associated with the Chongqing Han population of diabetic patients and serum GAD-Ab levels. For the A allele genotype, the AA and AA + AG genotypes were correlated with a significant increase in GAD-Ab-positive results.

This study was limited to the Chongqing population; SNPs often show ethnic and regional differences. Thus, the association of GAD2 5'-UTR polymorphic loci with susceptibility to T2DM and the relationship of rs2236418 with T2DM in patients with GAD-Ab should be examined in a larger population and including other ethnic groups. Gene polymorphisms and T2DM-related mechanisms must also be further examined.

\section{Conflicts of interest}

The authors declare no conflict of interest.

\section{REFERENCES}

Ali F, Rowley M, Jayakrishnan B, Teuber S, et al. (2011). Stiff-person syndrome (SPS) and anti-GAD-related CNS degenerations: protean additions to the autoimmune central neuropathies. J. Autoimmun. 37: 79-87.

Appel SJ, Wadas TM, Rosenthal RS and Ovalle F (2009). Latent autoimmune diabetes of adulthood (LADA): An often misdiagnosed type of diabetes mellitus. J. Am. Acad. Nurse Pract. 21: 156-159.

Boutin P, Dina C, Vasseur F, Dubois S, et al. (2003). GAD2 on chromosome 10p12 is a candidate gene for human obesity. PLoS Biol. 1: E68. 
Brooks-Worrell BM, Reichow JL, Goel A, Ismail H, et al. (2011). Identification of autoantibody-negative autoimmune type 2 diabetic patients. Diabetes Care 34: 168-173.

Davies H, Brophy S, Fielding A, Bingley P, et al. (2008). Latent autoimmune diabetes in adults (LADA) in South Wales: incidence and characterization. Diabet. Med. 25: 1354-1357.

Genuth S, Alberti KG, Bennett P, Buse J, et al. (2003). Follow-up report on the diagnosis of diabetes mellitus. Diabetes Care 26: 3160-3167.

Hampe CS, Hall TR, Agren A and Rolandsson O (2007). Longitudinal changes in epitope recognition of autoantibodies against glutamate decarboxylase $65(\mathrm{GAD} 65 \mathrm{Ab})$ in prediabetic adults developing diabetes. Clin. Exp. Immunol. 148: $72-78$.

Hansen KB, Vilsbøll T and Knop FK (2010). Incretin mimetics: a novel therapeutic option for patients with type 2 diabetes - a review. Diabetes Metab. Syndr. Obes. 3: 155-163.

Jayakrishnan B, Hoke DE, Langendorf CG, Buckle AM, et al. (2011). An analysis of the cross-reactivity of autoantibodies to GAD65 and GAD67 in diabetes. PLoS One 6: e18411.

Johnson GC, Payne F, Nutland S, Stevens H, et al. (2002). A comprehensive, statistically powered analysis of GAD2 in type 1 diabetes. Diabetes 51: 2866-2870.

Jun HS, Chung YH, Han J, Kim A, et al. (2002). Prevention of autoimmune diabetes by immunogene therapy using recombinant vaccinia virus expressing glutamic acid decarboxylase. Diabetologia 45: 668-676.

Kadiyala R, Peter R and Okosieme OE (2010). Thyroid dysfunction in patients with diabetes: clinical implications and screening strategies. Int. J. Clin. Prasct. 64: 1130-1139

Karlsen AE, Hagopian WA, Grubin CE, Dube S, et al. (1991). Cloning and primary structure of a human islet isoform of glutamic acid decarboxylase from chromosome 10. Proc. Natl. Acad. Sci. U S A 88: 8337-8341.

Kaufman DL, Clare-Salzler M, Tian J, Forsthuber T, et al. (1993). Spontaneous loss of T-cell tolerance to glutamic acid decarboxylase in murine insulin-dependent diabetes. Nature 366: 69-72.

Li H, Gan W, Lu L, Dong X, et al. (2013). A genome-wide association study identifies GRK5 and RASGRP1 as type 2 diabetes loci in Chinese Hans. Diabetes 62: 291-298.

Prokopenko I, McCarthy MI and Lindgren CM (2008). Type 2 diabetes: new genes, new understanding. Trends Genet. 24: 613-621.

Scott LJ, Mohlke KL, Bonnycastle LL, Willer CJ, et al. (2007). A genome-wide association study of type 2 diabetes in Finns detects multiple susceptibility variants. Science 316: 1341-1345.

Sosenko JM, Skyler JS, Palmer JP, Krischer JP, et al. (2011). A longitudinal study of GAD65 and ICA512 Autoantibodies during the progression to type 1 diabetes in diabetes Prevention Trial-Type 1(DPT-1) participants. Diabetes Care 34: 2435-2437.

Wheeler E and Barroso I (2011). Genome-wide association studies and type 2 diabetes. Brief. Funct. Genomics 10: 52-60.

Yasuda K, Miyake K, Horikawa Y, Hara K, et al. (2008). Variants in KCNQ1 are associated with susceptibility to type 2 diabetes mellitus. Nat. Genet. 40: 1092-1097. 